\title{
Visco-thermo-elastic Simulation Approach For Prediction of Cure-induced Residual Stresses in Fiber Reinforced Composites
}

\author{
Jonas Müller, Michael Müller-Pabel, Niklas Lorenz, Benjamin Gröger, Johannes Gerritzen, Maik Gude and Christian
} Hopmann

\author{
Jonas Müller. Institute for Plastics Processing, RWTH Aachen, Seffenter Weg 201, 52074 Aachen. \\ Michael Müller-Pabel. Institute of Lightweight Engineering and Polymer Technology, TU Dresden, Holbeinstraße 3, 01307 \\ Dresden. \\ Corresponding author: Michael Müller-Pabel. E-mail address: michael.mueller-pabel@tu-dresden.de \\ Niklas Lorenz. Institute for Plastics Processing, RWTH Aachen, Seffenter Weg 201, 52074 Aachen. \\ Benjamin Gröger. Institute of Lightweight Engineering and Polymer Technology, TU Dresden, Holbeinstraße 3, 01307 Dresden. \\ Johannes Gerritzen. Institute of Lightweight Engineering and Polymer Technology, TU Dresden, Holbeinstraße 3, 01307 Dresden. \\ Maik Gude. Institute of Lightweight Engineering and Polymer Technology, TU Dresden, Holbeinstraße 3, 01307 Dresden. \\ Christian Hopmann. Institute for Plastics Processing, RWTH Aachen, Seffenter Weg 201, 52074 Aachen.
}

\begin{abstract}
Liquid composite molding (LCM) has established as a high quality manufacturing process for fiber reinforced composite structures. In order to reduce cycle times significantly, novel fast curing matrix resins are being introduced into series production. These put high requirements on process control and part reproducibility. Problems that may be encountered in this context involve process-induced distortion and surface waviness resulting from anisotropic and cure-dependent material properties. Numerical simulations represent a powerful approach to avoid the use of costly trial-and-error methods. For this reason, a simulation approach is being developed which aims at the prediction of residual stresses and accompanying effects on different length scales. Based on a resin characterization comprising reaction kinetics, cure-dependent relaxation modulus as well as thermal expansion and pressure-dependent chemical shrinkage, a generalized MAXWELL model is selected to describe the process-related mechanical behavior of the thermoset. Taking into account the influence of the process parameters on the resin properties enables a detailed analysis of processproperty-relationships. By this, the developed simulation approach offers the possibility of a comprehensive analysis of both local and global process-induced phenomena and hence prevention of flaws.
\end{abstract}

Keywords. Cure Dependence, Numerical Modelling, Experimental

\section{Introduction}

The future use of fiber reinforced polymers (FRP) for series production of lightweight structures primarily depends on availability of competitive, cost-efficient and reproducible manufacturing technologies. Liquid composite molding (LCM) represents a well-established production route, which nevertheless suffers from certain drawbacks associated with the complex FRP material behavior. Especially the interaction of fiber and matrix as well as the cure-dependent resin properties represent major challenges for part design and process control. As the thermo-mechanical behavior of the resin depends on a number of process parameters like time, temperature, degree of cure and pressure, material characterization and modelling require a comprehensive strategy.

Previously published work on cure-dependent resin behavior mainly focused on better understanding of conventional prepreg and RTM manufacturing processes [1-4]. Only few attempts were made to analyze the process-dependent properties of fast curing resins [5,6]. Furthermore, pressure dependence is often neglected, which is questionable when it comes to newly developed technologies with high impregnation speed like high pressure RTM (HP-RTM) or wet compression molding [7]. To fill this gap, an appropriate material model approach in combination with reliable material data are required. For this purpose, a visco-thermo- elastic simulation approach is selected [8] and 
Visco-thermo-elastic Simulation Approach For Prediction of Cure-induced Residual Stress...

calibrated. Cure-dependent properties were determined by shear rheological and dynamic-mechanical analyses (DMA) on partially cured neat resin specimens. A newly developed volumetric dilatometer is used to analyze pressure- und cure-dependent chemical shrinkage at process-related conditions [9]. The determined relationships between resin behavior and process parameters are transferred to the material model and used for simulations on micro-scale aiming at reproduction of surface waviness effects and residual stresses.

\section{Methods}

\subsection{Experimental set up}

The experimental program described here is complementary to a previously published work of the authors [10]. In this contribution, special attention is paid to pressure-dependent chemical shrinkage and cure-dependence of viscothermo-elastic modulus of the fast curing resin system TRAC Epikote 06150, which is used for RTM processes in automotive industry. Isothermal $\left(80,90,100\right.$ and $\left.120^{\circ} \mathrm{C}\right)$ measurements at different pressure loads $(5,10,25$ and 60 bar) are carried out using a dilatometer optimized for the characterization of fast curing resin systems [2,9]. Samples from the cured dilatometer specimens are prepared for the subsequent DSC measurement. The DSC analysis is conducted using the Q2000 device from TA Instruments Inc., Austin, USA, according to the specifications described in DIN 65467. A heating and cooling rate of $10 \mathrm{~K} / \mathrm{min}$ is selected for all measurements and applied to a temperature range from $-50{ }^{\circ} \mathrm{C}$ to $250^{\circ} \mathrm{C}$. The degree of cure (DOC) $\xi$ is determined from the value of remaining reaction enthalpy $\Delta H_{R}$ and the total reaction enthalpy $\Delta H_{R, \text { total }}$ according to eq. 1 [11]:

$$
\xi=1-\frac{\Delta H_{R}}{\Delta H_{R, t o t a l}}
$$

The cure-dependent visco-thermo-elastic properties were determined using an Anton Paar MCR 502 rheometer device. A plateplate measurement system with a diameter of $25 \mathrm{~mm}$ and a gap of $1.5 \mathrm{~mm}$ was used to measure in the liquid stage as well as the liquid-solid transition during gelation, which was determined to occur at $\xi=0.68$. To allow continuous measurements, oscillation mode at a frequency of $1 \mathrm{~Hz}$ and a shear deformation amplitude of $1 \%$ were chosen. Isothermal temperatures of $60^{\circ} \mathrm{C}, 80^{\circ} \mathrm{C}, 100{ }^{\circ} \mathrm{C}$ and $120^{\circ} \mathrm{C}$ were used. The data are transferred from time scale to DOC scale by using the previously published reaction kinetics model of the resin system [10].

In order to cover the entire post-gelation area, a solid rectangular fixture (SRF) was used to analyze the influence of varying DOCs using specimens with a cross section of $2 \mathrm{~mm} \times 4 \mathrm{~mm}$ and a length of $40 \mathrm{~mm}$. In order to obtain time-dependent modulus data, relaxation experiments with an initial shear deformation of $0.1 \%$ were performed at $5 \mathrm{~K}$ temperature intervals ranging from room temperature to a temperature $10 \mathrm{~K}$ below the actual glass transition. A relaxation time of $5 \mathrm{~min}$ was required to generate overlapping stiffness values between the selected temperature segments. Partial cure was realized by using a pre-cure schedule of $7 \mathrm{~h}$ at $60{ }^{\circ} \mathrm{C}$ within a molding tool; followed by progressive post-cure schedules according to Table 1 that were performed in a convection oven. 
Table 1. Post-cure cycles for generation of SRF specimens with graded DOC.

\begin{tabular}{llll}
\hline Post-cure temperature $\left({ }^{\circ} \mathrm{C}\right)$ & Dwell time $(\mathrm{min})$ & Resulting $\mathrm{Tg}\left({ }^{\circ} \mathrm{C}\right)$ & Corresponding DOC (-) \\
\hline- & - & 80 & 0.88 \\
\hline 75 & 10 & 87 & 0.91 \\
\hline 85 & 10 & 99 & 0.95 \\
\hline 95 & 10 & 110 & 0.96 \\
\hline 105 & 10 & 120 & 0.97 \\
\hline 130 & 10 & 123 & 1 \\
\hline
\end{tabular}

\subsection{Material modelling approach}

For simulating cure-dependent material behavior, a material model displaying the process-dependence is crucial. Based on the findings from Müller et al. [10] for fully cured resin, a material model is developed to model cure-dependent material behavior during the manufacturing process. In this study, the epoxy resin was modelled by a cure-dependent viscoelastic constitutive model developed and implemented as UMAT for ABAQUS based on the nonlinear viscoelastic constitutive model by Brandt and Rozo Lopez [8,12]. The cure-dependent viscoelastic behavior is modelled by a generalized MAXWELL model, taking into account temperature influence by a time/temperature shift function based on a modified WILLIAMS-LENDEL-FERRY (WLF)-approach [13]. The model presented allows to display effects of curedependent visco-thermo-elasticity, cure and pressure dependent volumetric shrinkage as well as thermal expansion. Thermal expansion and chemical shrinkage are implemented by using UEXPAN.

In order to calculate the cure state $\xi$, a $n^{\text {th }}$-order and an autocatalytic type was selected (eq. 2 and 3):

$$
\begin{gathered}
\frac{d \xi}{d t}=k_{1} \xi^{m}(1-\xi)^{n_{1}}+k_{2}(1-\xi)^{n_{2}} \text { and } \\
k_{i}=A_{i} e^{-E_{i} / R T}
\end{gathered}
$$

with $A_{i}, n_{i}, m, E_{i}$ being material parameters and $R$ the universal gas constant defined in [10]

$$
T_{g}(\xi)=\frac{\lambda \cdot \xi}{1-(1-\lambda) \xi}\left(T_{g \infty}-T_{g 0}\right)+T_{g 0}
$$

with $T_{g 0}, T_{\mathrm{g} \infty}, \lambda$ being material parameters determined by DSC scans on partially cured resin material and linear regression. Values are listed in Table 2.

Table 2. Parameter of DiBenedetto equation. 
Visco-thermo-elastic Simulation Approach For Prediction of Cure-induced Residual Stress...

\begin{tabular}{ccc}
\hline$\lambda(-)$ & $T_{g^{\infty}}\left({ }^{\circ} \mathrm{C}\right)$ & $T_{g 0}\left({ }^{\circ} \mathrm{C}\right)$ \\
\hline 0.396 & 126.10 & -37.65 \\
\hline
\end{tabular}

During curing of the resin, mechanical properties are steadily changing due to network growth, gelation and vitrification. To model the cure-dependent relaxation curves extracted from experiments, in a first step Prony series is fitted to generated master curves for $\xi=1$ according to eq. 5 [10]. $k$ matches the $k^{\text {th }}$ experimental master curve, while $k=1$ corresponds to the master curve at $\xi=1$ :

$$
G^{k=1}(t)=G_{0}^{k=1}\left(1-\sum_{i=1}^{n} g_{i}^{k=1}\left(1-e^{-t / \tau_{i}^{k=1}}\right)\right)
$$

$n$ is determined by fit with lowest residual mean square error (RMSE). Generally, 1 to 15 Prony elements are tested. According to O’Brien et al. [15] equilibrium shear modulus $G_{\infty}$ can be calculated by eq. 6 :

$$
\log \left(G_{\infty}\right)=C_{G_{\infty}}+\frac{D_{G_{\infty}}}{1+\exp \left(\frac{\xi_{g e l}^{-\xi}}{F_{G_{\infty}}}\right)}
$$

where $C_{\mathrm{g} \infty}, D_{\mathrm{g} \infty}$ and $F_{\mathrm{g} \infty}$ are material parameters and $\xi_{\text {gel }}$ represents the degree of cure at gelation.

For fitting the remaining master curves, following workflow is used:

- Hence relaxation data at lower $\xi$ is unknown, data for high relaxation times is augmented following eq. 6 for $t>10^{13} s$ - Prony series is fitted with $n$ equal to $n$ for $k=1$

- Equilibrium shear stiffness is used for calculation of $G(t, \xi)$.

Based on these Prony fits, $G(t)$ for $\xi^{\mathrm{k}+1} \leq \xi \leq \xi^{\mathrm{k}}$ is interpolated. For $\xi<\xi^{\max (k)}$ eq. 8 is used to calculate time-dependent shear modulus:

$$
\begin{aligned}
G_{0}(\xi) & =\frac{G_{\infty}(\xi)}{1-\Sigma_{i=1}^{n} g_{i}^{\max (k)}} \\
G(t, \xi) & =G_{0}(\xi) \cdot \frac{G^{\max (k)}(t)}{G_{0}^{\max (k)}}
\end{aligned}
$$

Temperature- and cure-dependence are considered by shift factor $a_{T}$ which is calculated by a modified WLF-approach [13]:

$$
\log \left(a_{T}\right)=C_{1}\left(\frac{T-T_{g}}{C_{2}+\left|T-T_{g}\right|}-\frac{T_{0}-T_{g}}{C_{2}+\left|T_{0}-T_{g}\right|}\right)
$$


$C_{1}, C_{2}$ are experimentally determined parameters. $T_{0}$ is the selected reference temperature of $40^{\circ} \mathrm{C} . t$ is substituted by reduced time $t_{\text {red }}$ following eq. 10 :

$$
t_{\text {red }}=a_{T} \cdot t
$$

Due to the use of UEXPAN for thermal expansion and chemical shrinkage, providing thermal strain increment $\triangle$ EUEXPAN $^{\text {th }}$ within UEXPAN is necessary. Generally chemical volume change $d V_{c h}$ is expressed by chemical strain increment $\Delta \varepsilon_{c h}$ assuming a cube with side length $l$ as followed:

$$
\begin{aligned}
d V_{c h} & =\frac{l^{3}-\left(l+d \varepsilon_{c h} l\right)^{3}}{l^{3}} \\
d \varepsilon_{c h} & =\left(1-d V_{c h}\right)^{1 / 3}-1 \\
\Delta \varepsilon_{c h} & =\left(1-\Delta V_{c h}\right)^{1 / 3}-1
\end{aligned}
$$

Assuming that the volumetric shrinkage is proportional to $\xi$ at a constant pressure $p$ leads to:

$$
d V_{c h}=f(p) d \xi \stackrel{\Delta}{\rightarrow} \Delta V_{c h}=f(p) \Delta \xi
$$

where $f(p)$ is an experimentally determined function (cf. eq. 17).

Following this, $\triangle E U E X P A N^{\text {th }}$ is equal to the sum of actual thermal expansion increment $\Delta \varepsilon$ th and chemical shrinkage increment $\Delta \varepsilon_{c h}$ :

$$
\Delta \varepsilon_{U E X P A N}^{t h}=\Delta \varepsilon_{t h}+\Delta \varepsilon_{c h}
$$

$\Delta \varepsilon t h$ is calculated as followed, where thermal expansion coefficient $\alpha_{t h}$ is assumed as constant defined in [10]:

$$
\Delta \varepsilon_{t h}=\alpha_{t h} \Delta T
$$

\subsection{Simulation approach}

The material model described above, was used in two different simulation models. The first model consists of a cube of unit length, which is subjected to pure shear in form of a unit jump within $0.5 \mathrm{sec}$. Afterwards the shear deformation is held constant for $300 s$ to reproduce the relaxation experiments. Throughout simulation time, the model is subjected to constant temperature. The unit length cube model is used to verify the above shown methodology for determining cure-dependent visco-thermo-elasticity.

The second simulation model represents a representative volume element (RVE) consisting of matrix material and randomly distributed glass fibers with a diameter of $7 \mu \mathrm{m}$ and a fiber volume fraction of $45 \%$ according to [10]. The 
Visco-thermo-elastic Simulation Approach For Prediction of Cure-induced Residual Stress...

overall cross section in undeformed state has dimensions of $50 \mu m \times 75 \mu m$. The model thickness is $1 \mu m$. Periodic boundary conditions are assigned to the lower surface, while the upper layer represents the component surface. The RVE is used for virtual parametric studies. These include the application of different material models (visco-thermo elastic and cure-dependent visco-thermo elastic) and the variation of the surface boundary conditions during the cooling phase (fix, free). In total 22,715 elements (C3D8 and C3D6) are used.

\section{Results}

\subsection{Experimental Results}

The results of volumetric dilatometer and DSC measurements are depicted in Figure 1. According to previous studies a linear approach was chosen to model the development of the chemical shrinkage after gelation $[2,16]$. For gelation time and corresponding DOC values the mean value of $\xi=0.68$ was used according to [10].

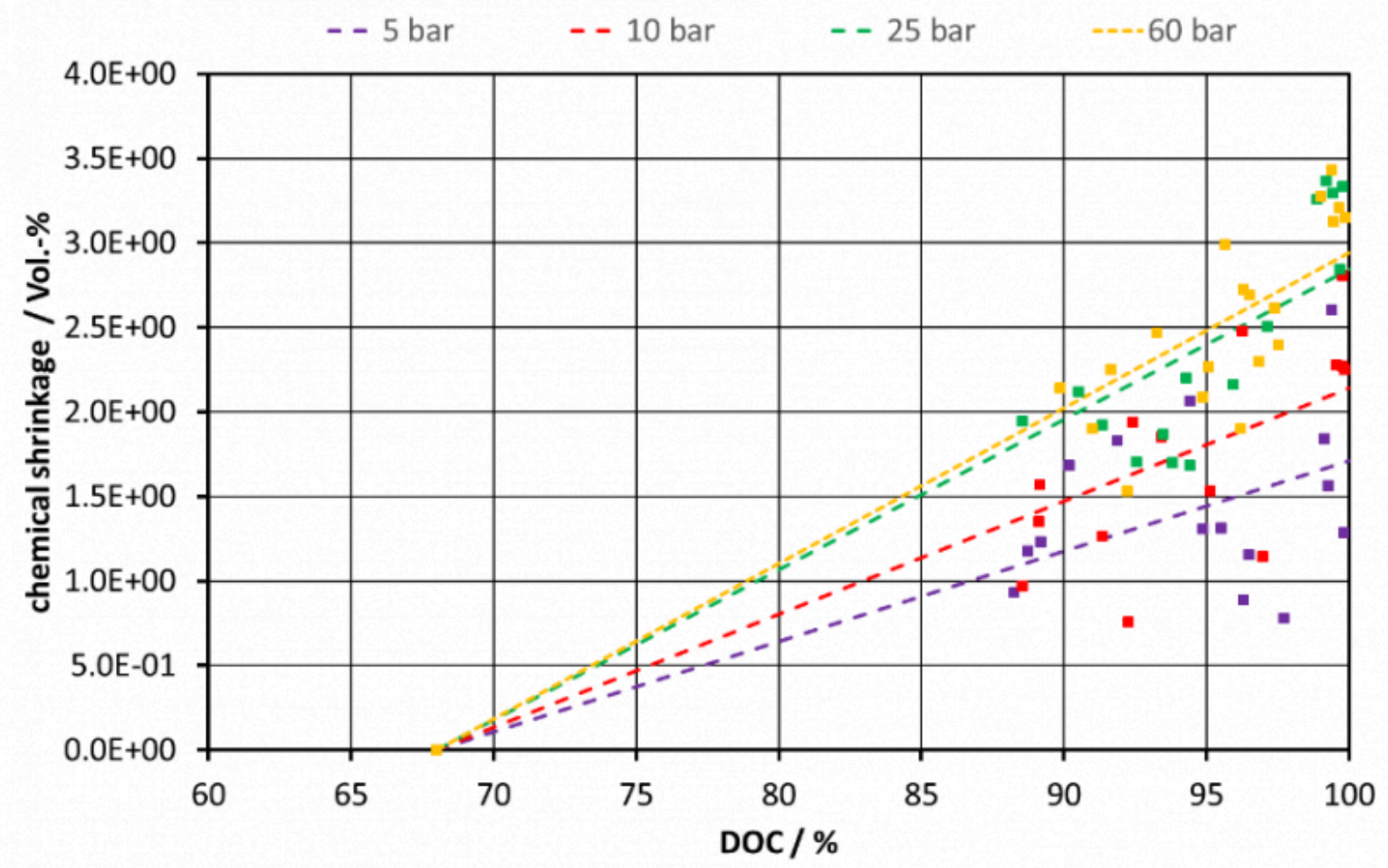

Figure 1:Pressure dependent chemical shrinkage and corresponding DOC values

Considering eq. 14 the pressure dependence of the chemical volume shrinkage can be fitted by eq. 17 and the data contained in Figure 1. For high pressure load eq. 17 approaches a limiting value.

$$
f(p)=a_{1} \arctan \left(a_{2} p\right)+a_{3}
$$

With $a_{1}, a_{2}$, $a_{3}$ representing fitting parameters and values listed in Table 3. 
Table 3. Fitting parameters

\begin{tabular}{lll}
\hline$a_{1}(-)$ & $a_{2}\left(\right.$ bar $\left.^{-1}\right)$ & $a_{3}(-)$ \\
\hline 0.068 & 0.253 & -0.009 \\
\hline
\end{tabular}

Thus, elevated pressure loads result in increasing chemical volume shrinkage. Nawab et al. [17] have observed an effect of the hydrostatic pressure on the resulting chemical volumetric shrinkage of a neat resin sample. They justify the increase in chemical shrinkage by a decreasing free volume due to the high-pressure loads applied [17]. Li et al. [18] proved by means of molecular dynamic simulations that the free volume fraction increases during the curing process [18]. As the free volume fraction reduces with high pressure loads an increasing change of the volume can be expected with elevated curing pressures [19].

In order to provide experimental reference data for definition of the Prony parameters, cure-dependent data for the instantaneous modulus $G_{0}$ and the equilibrium modulus $G_{\infty}$ as well as the relaxation behavior between glassy and rubbery regime are required. While the determination of $G_{0}$ can be realized straightforward by evaluating the initial stress response of the relaxation experiments at low temperatures, the determination of $G_{\infty}$ is more challenging due to possible post-cure when using partially cross-linked specimens. For this reason, values for $G_{\infty}$ at $\xi<1$ are taken from the isothermal oscillating measurements in plate-plate mode. The evaluation of these experiments is limited to a DOC of 0.85 , as in plate-plate-mode the increasing resin stiffness leads to residual stresses in combination with hindered lateral contraction. Consequently, a relaxation experiment on a fully cured specimen is used to generate an additional value for $G_{\infty}$ at $\xi=1$. The results are shown in Figure 2 together with the fit based on the work of [20] and according to eq. 6. Exact values for eq. 6 are listed in Table 4. 
Visco-thermo-elastic Simulation Approach For Prediction of Cure-induced Residual Stress...

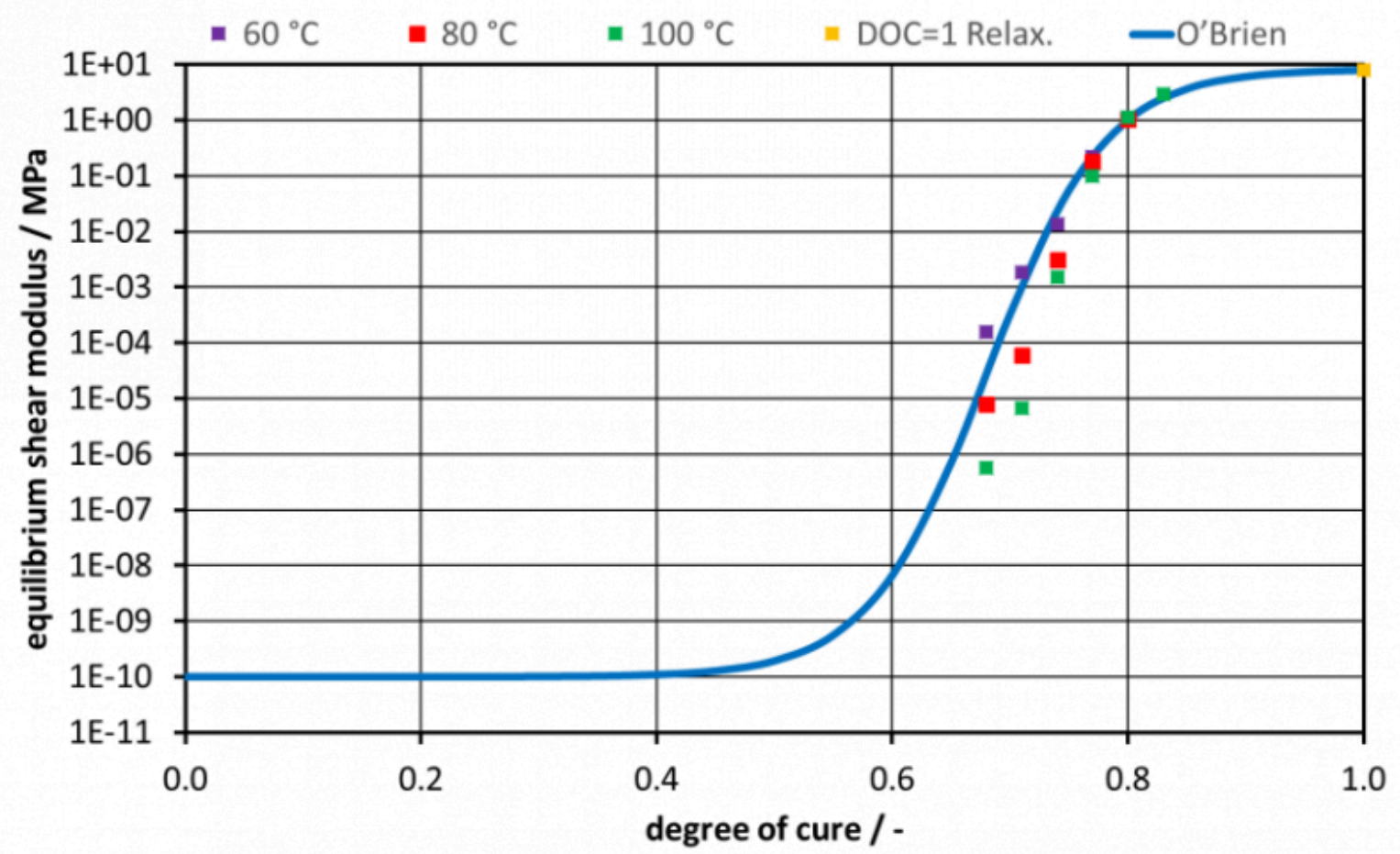

Figure 2: Comparison between experimental data and fit for equilibrium shear stiffness

Table 4. Parameters of O’Brien equation.

\begin{tabular}{lll}
\hline$C_{G}(\log (M P a))$ & $D_{G}(\log (M P a))$ & $F_{G}(-)$ \\
\hline-10 & 10.92 & 0.05 \\
\hline
\end{tabular}

The cure- and time-dependent relaxation behavior of the resin system is evaluated by using the time-temperature superposition principle. Individual master curves are constructed for the DOC from 0.88 to 1 at a reference temperature of $40{ }^{\circ} \mathrm{C}$. The results with corresponding Prony series fit with $n=13$ following eq. 5 are shown in Figure 3. During fitting a RMSE of 0.001 was achieved. The derived Prony series are in good agreement with the experimental data. When plotting the master curves on the reduced time scale, it can be seen that the relaxation time increases with cure and the instantaneous modulus $G_{0}$ decreases with cure, which can be attributed to reduced mobility during cooling at higher degrees of cure [21].

Figure 4 shows shift factors and the corresponding fits following eq. 9. Generally, the slope of time-temperature shift factors $\log \left(a_{T}\right)$ decrease with cure. Furthermore, to obtain good fitting results the parameters $C_{1}$ and $C_{2}$ are fitted individually. The individual Parameters can be found in Table 5. 


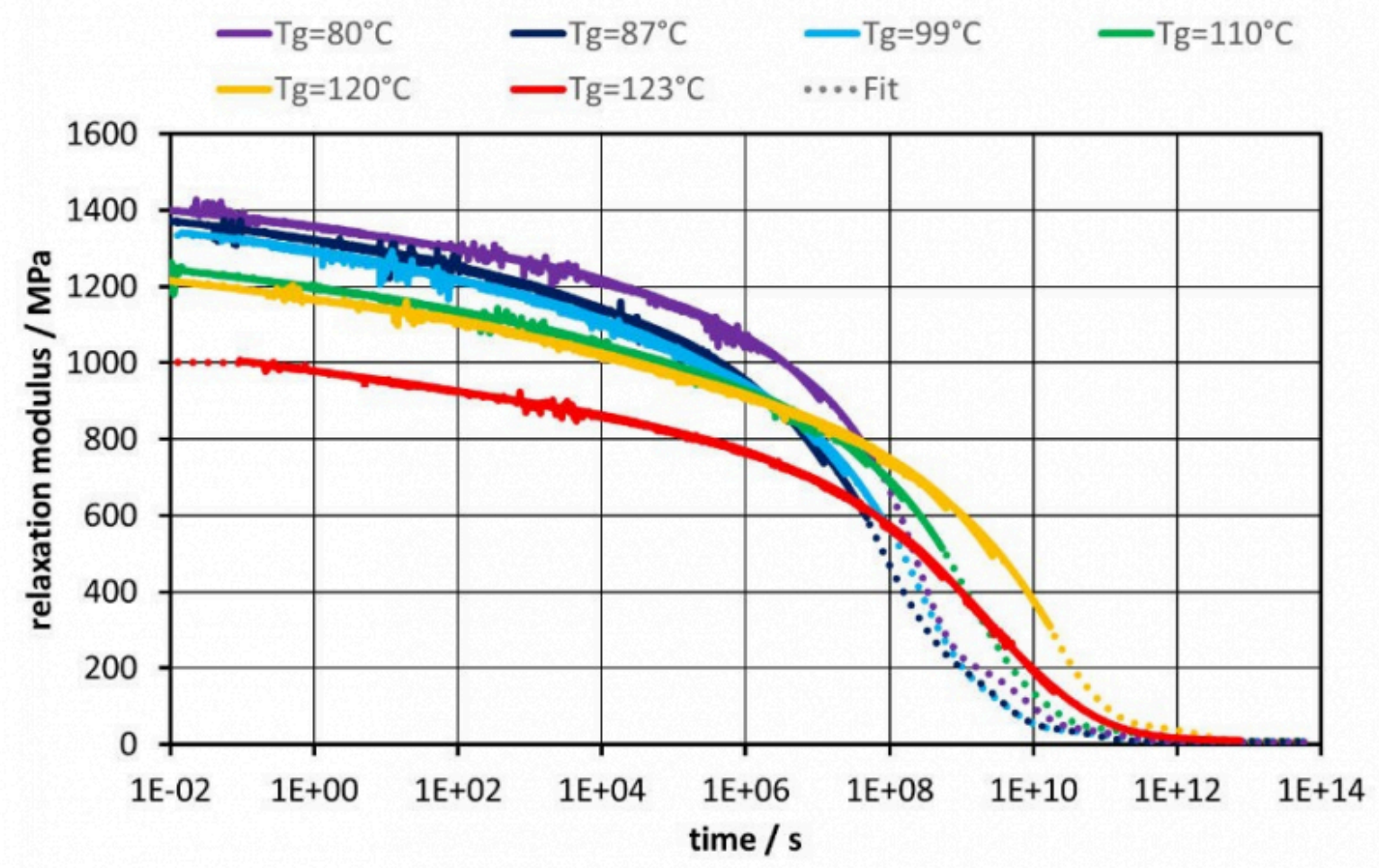

Figure 3: Prony series fit with $n=13$

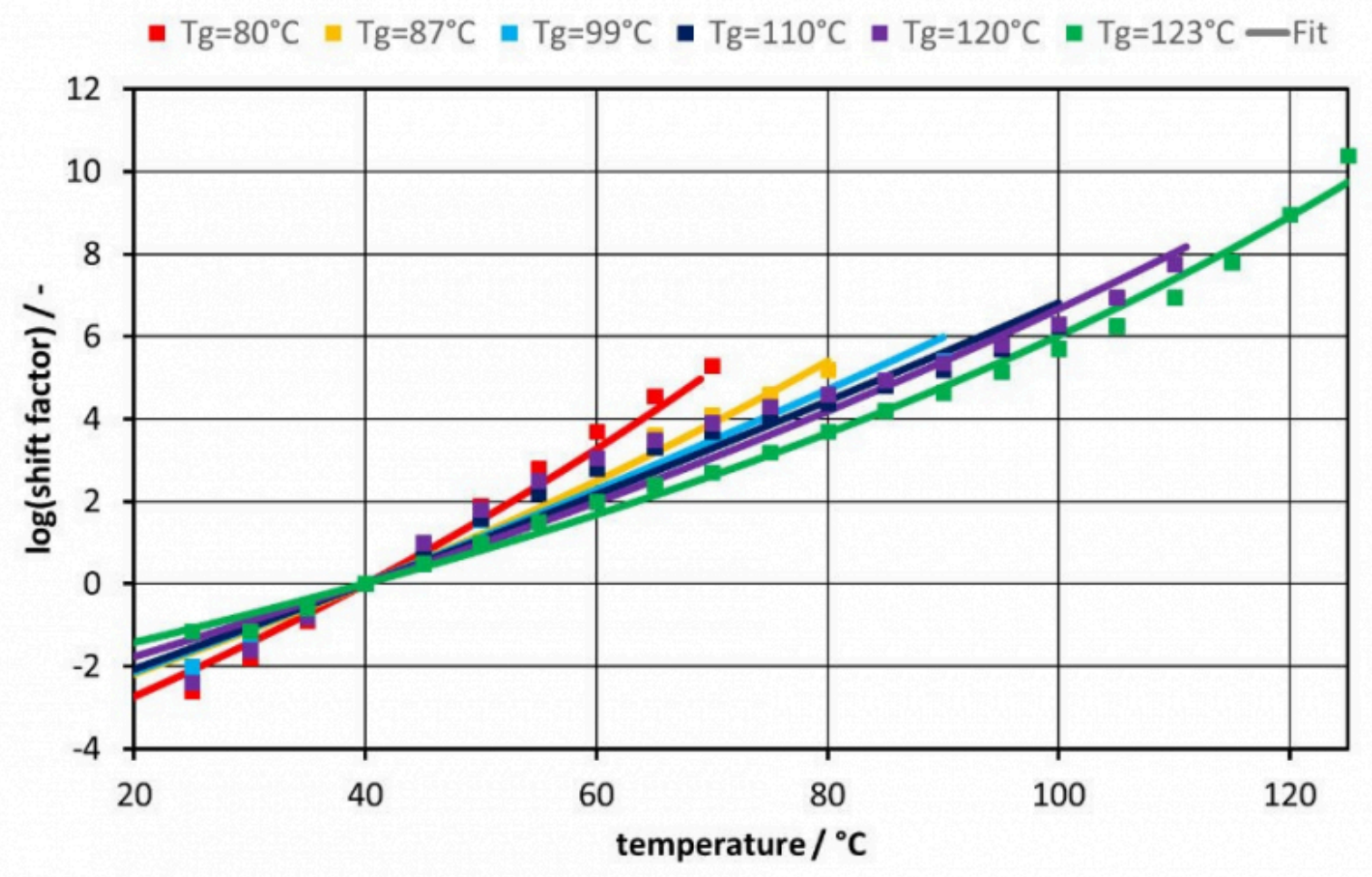


Visco-thermo-elastic Simulation Approach For Prediction of Cure-induced Residual Stress...

Figure 4: Comparison between shift factors from master curve generation and function fitted (cf. eq. 9)

Table 5. Parameters of eq. 9.

\begin{tabular}{lllllll}
\hline$T_{G}\left({ }^{\circ} \mathrm{C}\right)$ & 80 & 87 & 99 & 110 & 120 & 123 \\
\hline$C_{1}(-)$ & 40.0 & 40.0 & 80 & 100.0 & 45.0 & 29.81 \\
\hline$C_{2}\left({ }^{\circ} \mathrm{C}\right)$ & 180.0 & 240.0 & 600.0 & 800.0 & 300.0 & 175.33 \\
\hline
\end{tabular}

\subsection{Simulation results}

In a first step, a verification of the suggested approach was performed by using the unit length cube model. A set of simulations were executed in order to simulate time dependence of shear modulus. Therefore, simulations at different temperatures between $20^{\circ} \mathrm{C}$ and $130^{\circ} \mathrm{C}$ in $5 \mathrm{~K}$ steps and varying $\xi=\xi k$ were performed. Figure 5 shows exemplarily depicted results for $\xi=1.0$ in comparison to input data of the experimental relaxation tests. It shows that simulation data is in good agreement with experimental data and the material behavior is reproduced correctly.

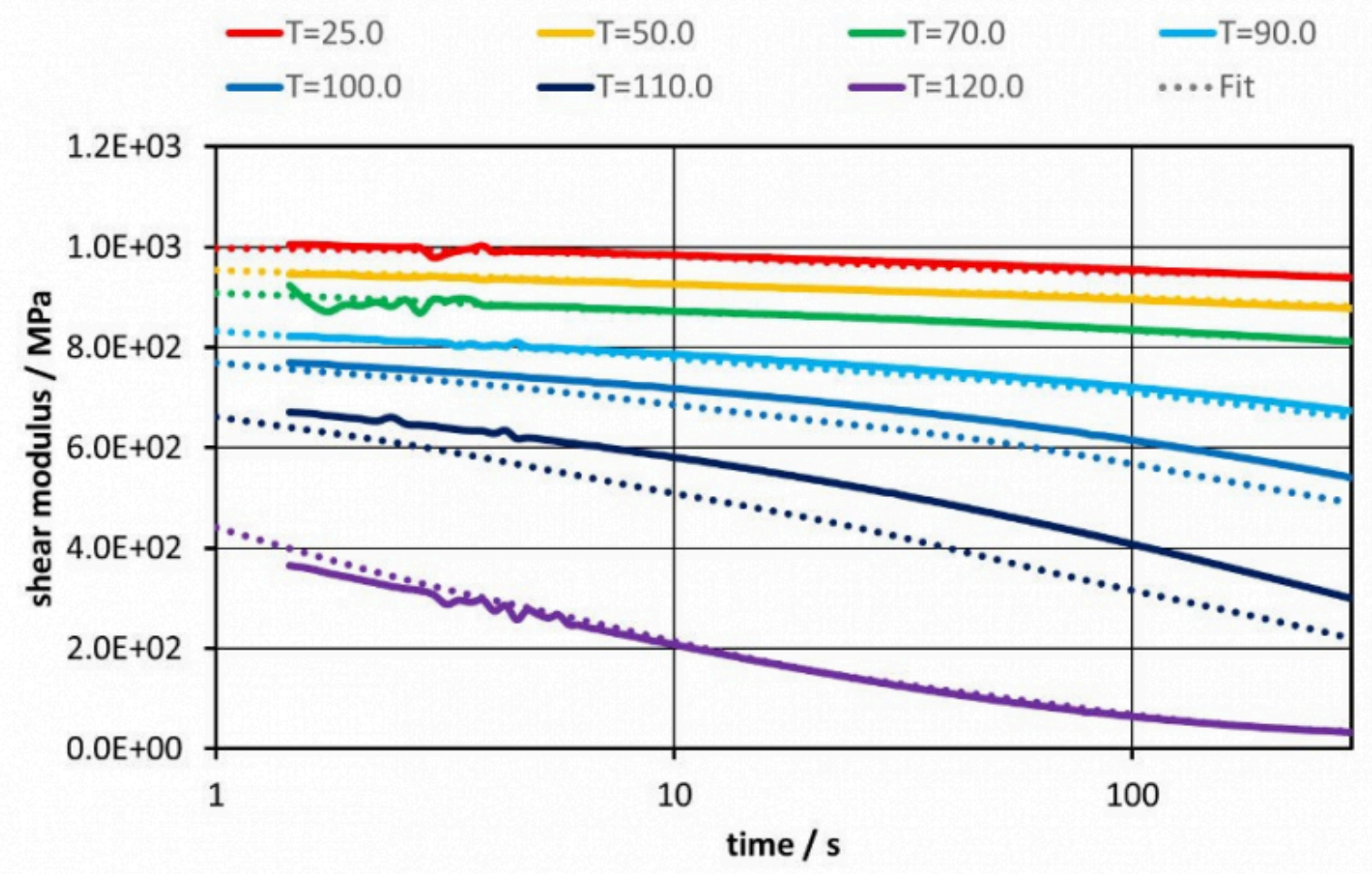

Figure 5: Comparison between input data and simulation data

In a second step, the material model presented was used to compute the displacement of the outer surface of a RVE after a generic cure cycle. The generic cure cycle consists of 4 phases (cf. Figure 6). First, the model is heated up from $60^{\circ} \mathrm{C}$ to $120^{\circ} \mathrm{C}$ with $20 \mathrm{~K} / \mathrm{min}$. In the second step, the model is subjected to a constant temperature of $120^{\circ} \mathrm{C}$ for 20 min in order 
to completely cure the resin. Afterwards, it is cooled down to $25^{\circ} \mathrm{C}$ with $-20 \mathrm{~K} / \mathrm{min}$. For a fixed top layer of the RVE the boundary is deleted which represents demolding, and the model is subjected to another 2 min at $25{ }^{\circ} \mathrm{C}$. For simulation in case of a free surface, surface is hindered in moving in positive 2-direction. Figure 6 shows the displacement of an exemplarily chosen node (1-coordinate $=25 \mu \mathrm{m}$ ) the upper surface over simulation time for an unconstrained surface. The evolution of displacement in thickness direction (U2) is mainly driven by chemical shrinkage during second stage and thermal expansion during cooling at third stage.

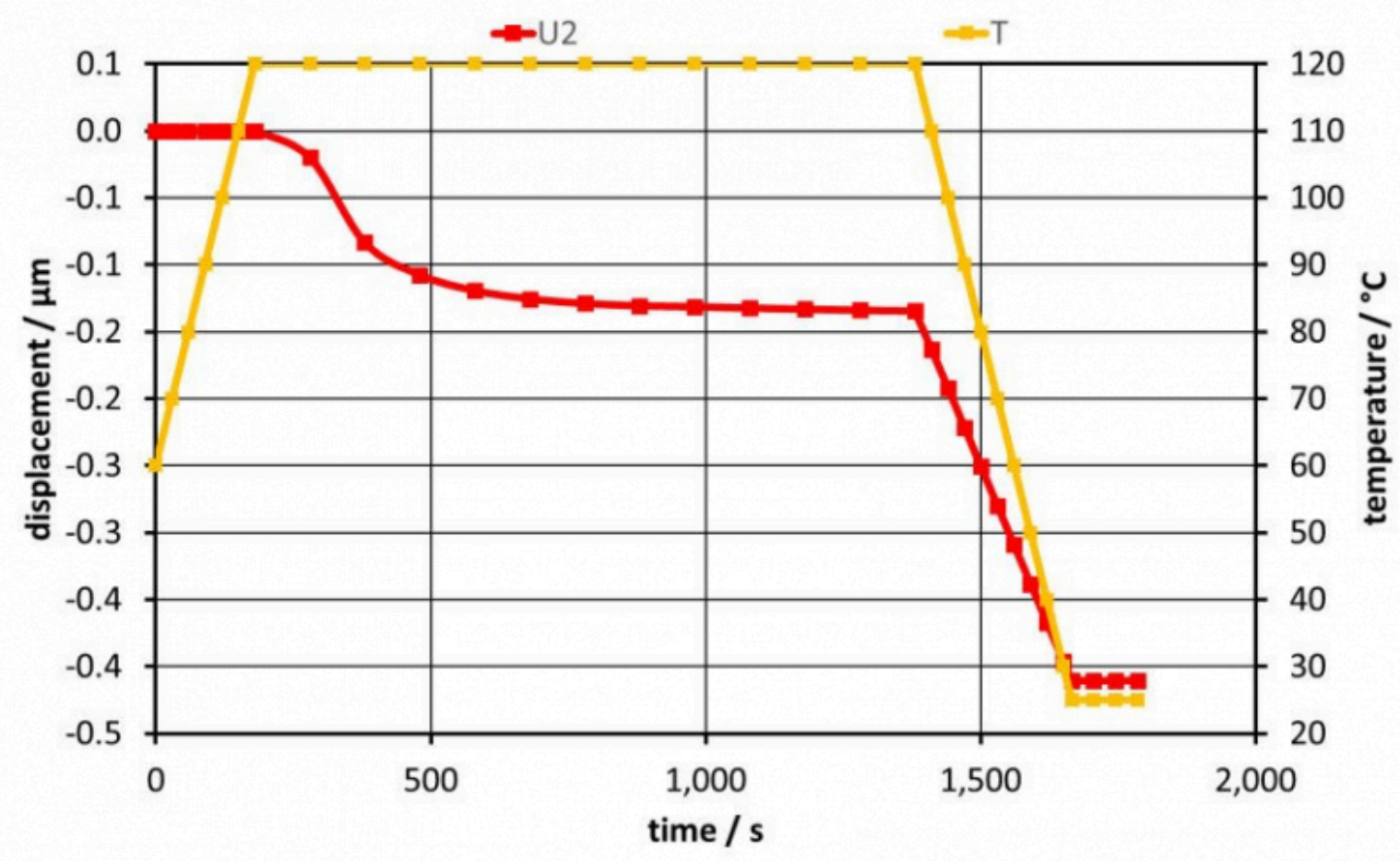

Figure 6: Displacement during cure of an exemplarily chosen node on the free surface

Figure 7 shows the deformation of the RVE using the cure-dependent visco-thermo-elastic material model. The results show that, regions with fewer fibers yield a higher deformation after completion of the cure cycle. 
Visco-thermo-elastic Simulation Approach For Prediction of Cure-induced Residual Stress...
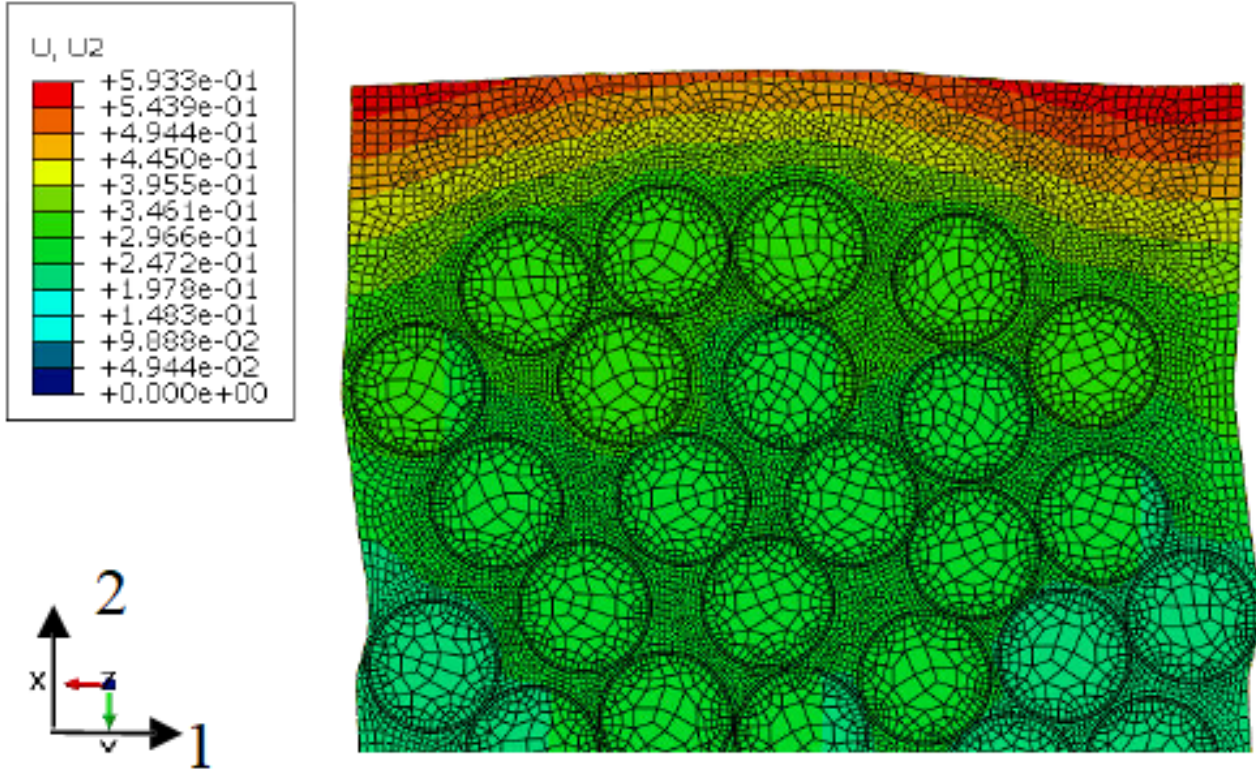

Figure 7: Deformed RVE (scale factor:10) calculated with cure-dependent visco-thermo-elastic matrix behavior and a free part surface during cooling

Figure 8 shows displacement in thickness direction of the top layer of the RVE for different material models and boundary conditions. The curves denoted with simple correspond to the visco-thermo-elastic material model from [10]. The model captures only time-dependent material behavior at fully cured stage during cool-down. In contrast, curves denoted with cure-dependent capture cure-dependent material behavior according to the suggested approach. This results mainly in additional contraction of the material due to chemical shrinkage and a different relaxation behavior at lower $\xi$.

The results for a fixed top layer differ only slightly, due to fixed surface and high relaxation at higher temperatures of residual stresses mainly induced by chemical shrinkage. Comparing the results to a free surface shows huge differences. This is based on chemical shrinkage due to unhindered deformation (cf. Figure 6). 
— cure dependent + fixed — cure dependent + free — simple + free- - simple + fixed

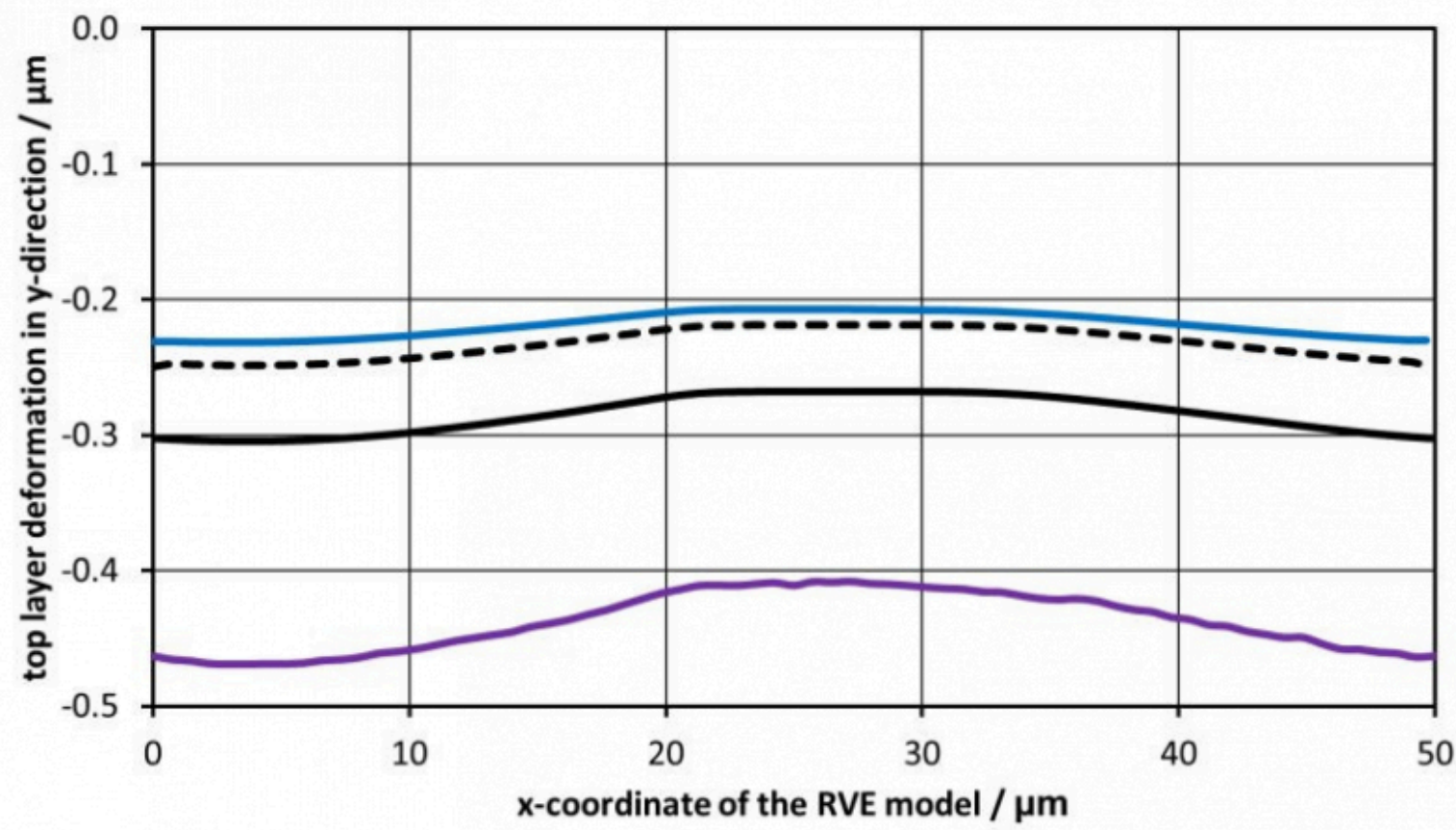

Figure 8: Displacement in thickness direction of top surface of RVE

\section{Conclusion}

The production of composite materials using fast curing resin systems has gained considerable interest in recent years. However, further advances in quality and reproducibility are required, especially for automotive applications. Component distortion and surface defects resulting from chemical shrinkage and thermal expansion pose major challenges that require costly quality control and process monitoring. The presented work aims at a comprehensive characterization and modeling of the underlying phenomena that lead to surface waviness and part distortions.

In this work, the material characterization of a fast curing epoxy resin based on [10] was extended by cure-dependent mechanical properties and pressure-dependent chemical shrinkage. The experimentally determined results were transferred into a material model to enable numerical studies of surface waviness and part distortion induced by manufacturing process. A RVE modeling method was proposed that allows sensitivity analysis.

In terms of verification, the developed cure-dependent viscoelastic material model shows good agreement with the relaxation test data. Regarding the selected RVE model, the visoc-elastic matrix behavior was shown to have only limited sensitivity to a change of surface the boundary condition. This does not apply to the cure-dependent viscothermo-elastic matrix behavior. In this case, the boundary condition during processing has a considerable influence on the resulting surface waviness. The assumption of a perfectly fixed top layer during cooling results in a significantly lower surface deformation compared to a free surface since deformations resulting from chemical shrinkage where hindered by the fixed top layer. Overall, the cure-dependent visco-thermo- elastic matrix behavior yields a maximum of deformation for an unconstrained surface.

Future work will focus on verification of the made assumptions and on modelling a multi-scale approach in order to 
Visco-thermo-elastic Simulation Approach For Prediction of Cure-induced Residual Stress...

determine surface waviness and part distortion on part level during curing.

\section{Acknowledgement}

This research is funded by the German Research Foundation (DFG) within the Project 415849481

\section{Bibliography}

[1] Ersoy N, Garstka T, Potter K, Wisnom MR, Porter D, Clegg M et al. Development of the properties of a carbon fibre reinforced thermosetting composite through cure. Composites Part A 2010;41(3):401-9. https://doi.org/10.1016/ j.compositesa.2009.11.007.

[2] Khoun L, Hubert P. Cure shrinkage characterization of an epoxy resin system by two in situ measurement methods. Polym. Compos. 2010;31(9):1603-10. https://doi.org/10.1002/pc.20949.

[3] Baran I, Cinar K, Ersoy N, Akkerman R, Hattel JH. A Review on the Mechanical Modeling of Composite Manufacturing Processes. Arch Computat Methods Eng 2017;24(2):365-95. https://doi.org/10.1007/s11831-016-9167-2.

[4] Zobeiry N, Forghani A, Li C, Gordnian K, Thorpe R, Vaziri R et al. Multiscale characterization and representation of composite materials during processing. Philos Trans A Math Phys Eng Sci 2016;374(2071):20150278. https://doi.org/ $10.1098 /$ rsta.2015.0278.

[5] Groh F, Kappel E, Hühne C, Brymerski W. Investigation of fast curing epoxy resins regarding process induced distortions of fibre reinforced composites. Composite Structures 2019;207:923-34. https://doi.org/10.1016/ j.compstruct.2018.09.003.

[6] Muc A, Romanowicz P, Chwał M. Description of the Resin Curing Process-Formulation and Optimization. Polymers (Basel) 2019;11(1). https://doi.org/10.3390/polym11010127.

[7] Keller A, Dransfeld C, Masania K. Flow and heat transfer during compression resin transfer moulding of highly reactive epoxies. Composites Part B: Engineering 2018;153:167-75. https://doi.org/10.1016/j.compositesb.2018.07.041.

[8] Rozo Lopez N, Chen J, Hopmann C. A micromechanical model for loading and unloading behavior of fiber reinforced plastics under cyclic loading. Polym Compos 2020;41(9):3892-902. https://doi.org/10.1002/pc.25684.

[9] Hopmann C, Wagner PN, Böttcher A. Development of a dilatometer for shrinkage analysis of thermosetting resin systems at accurate processing conditions of liquid composite moulding processes and exemplary results of the effects of varying processing conditions on chemical shrinkage of an epoxy resin. Journal of Composite Materials 2017;52(18):2451-61. https://doi.org/10.1177/0021998317747374.

[10] Müller M, Gerritzen J, Gröger B, Gude M. Thermomechanical Characterization of a Fast Curing Epoxy Resin for Simulation of Surface Waviness. Procedia Manufacturing 2020;47:129-33. https://doi.org/10.1016/ j.promfg.2020.04.152.

[11] Sibin Saseendran, Maciej Wysocki, Janis Varna. Cure-state dependent viscoelastic Poisson's ratio of LY5052 epoxy resin.

[12] Brandt M. CAE methods for the improved design of thermoplastic injection moulded parts. Dissertation. Aachen; 2006.

[13] Rowe GM, Sharrock MJ. Alternate Shift Factor Relationship for Describing Temperature Dependency of Viscoelastic 
Behavior of Asphalt Materials. Transportation Research Record 2011;2207(1):125-35. https://doi.org/10.3141/ 2207-16.

[14] Nielsen LE. Cross-Linking-Effect on Physical Properties of Polymers. Journal of Macromolecular Science, Part C: Polymer Reviews 1969;3(1):69-103. https://doi.org/10.1080/15583726908545897.

[15] Zarrelli M, Skordos AA, Partridge IK. Toward a constitutive model for cure-dependent modulus of a high temperature epoxy during the cure. European Polymer Journal 2010;46(8):1705-12. https://doi.org/10.1016/ j.eurpolymj.2010.06.002.

[16] Brauner C. Analysis of process-induced distortions and residual stresses of composite structures. Zugl.: Bremen, Univ., Diss., 2013. Berlin: Logos-Verl; 2013. [17] Nawab Y, Boyard N, Jaquemin F. Effect of pressure and reinforcement type on the volume chemical shrinkage in thermoset resin and composite. Journal of Composite Materials 2014;48(26):3191-9. https://doi.org/10.1177/0021998313502692.

[18] Li C, Strachan A. Free volume evolution in the process of epoxy curing and its effect on mechanical properties. Polymer 2016;97:456-64. https://doi.org/10.1016/j.polymer.2016.05.059.

[19] Deng Q Sundar CS, Jean YC. Pressure dependence of free-volume hole properties in an epoxy polymer. J. Phys. Chem. 1992(96):492-5.

[20] O'BRIEN DJ, MATHER PT, White SR. Viscoelastic Properties of an Epoxy Resin during Cure. Journal of Composite Materials 2001;35(10):883-904. https://doi.org/10.1177/a037323.

[21] White SR, Mather PT, Smith MJ. Characterization of the cure-state of DGEBA-DDS epoxy using ultrasonic, dynamic mechanical, and thermal probes. Polym Eng Sci 2002;42(1):51-67. https://doi.org/10.1002/pen.10927.

PDF automatically generated on 2021-05-20 17:31:55

Article url: https://popups.uliege.be/esaform21/index.php?id=4266

published by ULiège Library in Open Access under the terms and conditions of the CC-BY License (https://creativecommons.org/licenses/by/4.0) 